\title{
Pharmacokinetic Modeling and Prediction of Plasma Pyrrole-Imidazole Polyamide Concentration in Rats Using Simultaneous Urinary and Biliary Excretion Data
}

\author{
Takashi Nagashima, ${ }^{a}$ Takahiko Aoyama, ${ }^{a}$ Tsubasa Yokoe, ${ }^{a}$ Akiko Fukasawa, ${ }^{a}$ Noboru Fukuda, ${ }^{b}$ \\ Takahiro Ueno, ${ }^{c}$ Hiroshi SugiYama, ${ }^{d}$ Hiroki Nagase, ${ }^{b, e}$ and Yoshiaki Matsumoto ${ }^{*, a}$ \\ ${ }^{a}$ Department of Clinical Pharmacokinetics, College of Pharmacy, Nihon University; 7-7-1 Narashinodai, Funabashi, \\ Chiba 274-8555, Japan: ${ }^{b}$ Advanced Research Institute for the Science and Humanities, Nihon University; 4-2-1 Kudan- \\ kita, Chiyoda-ku, Tokyo 102-0073, Japan: ${ }^{c}$ Division of Nephrology and Endocrinology, Department of Medicine, Nihon \\ University School of Medicine; ${ }^{e}$ Division of Cancer Genetics, Department of Advanced Medical Science, Nihon University \\ School of Medicine; 30-1 Ooyaguchi-kami, Itabashi-ku, Tokyo 173-8610, Japan: and d Department of Chemistry, \\ Graduate School of Science, Kyoto University; Kitashirakawa, Oiwakecho, Sakyo-ku, Kyoto 606-8502, Japan. \\ Received December 19, 2008; accepted January 27, 2009
}

The use of urinary and/or biliary excretion data was considered as an alternative approach if the bioanalytical method lacked the appropriate sensitivity to adequately characterize the serum or plasma concentration-time profile. This approach is used for the analysis of plasma concentration-time profile under the lower limit of quantification (LLOQ) of various analytical instruments. The objective of this study was to develop a pharmacokinetic (PK) model that describes the plasma concentration-time profiles under LLOQ of HPLC using urinary and biliary excretion data. As model compounds, pyrrole (Py)-imidazole (Im) polyamides 1035 (MW, 1035.12) and 1666 (MW, 1665.78) were used. The cumulative urinary excretions of Py-Im polyamides 1035 and 1666 were $72.4 \pm 11.6$ and $4.8 \pm 0.5 \%$ of the administered dose, respectively. The cumulative biliary excretion of Py-Im polyamide 1035 was $4.3 \pm 0.4 \%$ of the administered dose, and Py-Im polyamide 1666 was not detected. The plasma concentration-time profiles of Py-Im polyamide 1035 were adequately described using linear and non-linear output compartments. The developed PK model could be used to describe the plasma concentration profiles using the linear output compartment interpreted as the urine compartment and the non-linear output compartment interpreted as the bile compartment. This PK model will be able to provide a more accurate prediction of the plasma concentration profiles under LLOQ.

Key words pharmacokinetics; modeling; pyrrole-imidazole polyamide; bile; urine; prediction

Pharmacokinetic (PK) modeling has been proposed as a means to improve the efficiency of drug development. It is difficult to develop an appropriate pharmacokinetic model if the lower limit of quantification (LLOQ) of the assay of the plasma drug concentration is high. The mathematical method of determining the plasma drug concentration below the LLOQ was discussed previously. ${ }^{1,2)}$ The method of analyzing the data including the urine concentration to construct the PK model that cannot be constructed using only plasma concentration data has also been reported. ${ }^{3)}$ Not only the plasma concentration data but also urine and bile data collected over time provided information that was used as basis for the development of a PK model. Although the use of serum or plasma concentration-time profiles was primarily recommended, the use of urinary and/or biliary excretion data was regarded as an alternative approach if the bioanalytical method lacked the appropriate sensitivity to adequately characterize the serum or plasma concentration-time profile. This approach is considered to be used for the analysis of plasma concentration-time profile under LLOQ of various analytical instruments.

The purpose of this study was to develop a PK model that describes the plasma concentration profiles under LLOQ of HPLC using urinary and biliary excretion data, and we used pyrrole (Py)-imidazole (Im) polyamides as model compounds.

\section{MATERIALS AND METHODS}

Chemicals and Reagents The Py-Im polyamides 1035 and 1666 reported previously ${ }^{4)}$ were purchased from Gentier Biosystems Co., Ltd. (Kyoto, Japan). Py-Im polyamide 1035 is composed of Ac-ImPyPy- $\gamma$-ImPyPy- $\beta$-Dp $(\beta$ : $\beta$-alanine, Dp: $N, N$-dimethylaminopropylamine, $\gamma: \gamma$-butyric acid), and Py-Im polyamide 1666 , with a higher molecular weight, is composed of Ac-PyPy- $\beta$-PyImPy- $\gamma$-PyPyPy- $\beta$-ImPy- $\beta$-Dp. The molecular weights of Py-Im polyamides 1035 and 1666 calculated on the basis of standard atomic weights ${ }^{5)}$ are 1035.12 and 1665.78, respectively. HPLC-grade methanol, acetonitrile, and distilled water were purchased from Kanto Chemical Co., Inc. (Tokyo, Japan). Midazolam and all other reagents of the highest quality were purchased from Wako Pure Chemical Industries, Ltd. (Tokyo, Japan). Pooled rat liver microsomes, pooled human liver microsomes, reduced nicotinamide adenine dinucleotide phosphate (NADPH) regenerating system solution, 1'-hydroxymidazolam, and 4-hydroxymidazolam were purchased from BD Bioscience (Woburn, MA, U.S.A.).

Analysis of Py-Im Polyamides Unchanged Py-Im polyamides 1035 and 1666 were extracted from rat urine and bile by solid-phase extraction using Oasis ${ }^{\circledR}$ WCX and MAX cartridges with both reverse-phase and ion-exchange sorbents. The HPLC method was based on a previous report with minor modification. ${ }^{4)}$ The quantitative analyses of PyIm polyamides 1035 and 1666 including the preparation procedure have been validated using the criteria of the Food and 
Table 1. Incubation Mixtures for Determining Metabolism of Py-Im Polyamides 1035 and 1666 by Liver Microsomes

\begin{tabular}{|c|c|c|c|c|}
\hline \multirow[b]{2}{*}{ Incubation mixture components } & \multicolumn{4}{|c|}{ Volume in incubation mixture $(\mu \mathrm{l})$} \\
\hline & Mix A & $\begin{array}{c}\text { Mix B } \\
\text { (blank 1) }\end{array}$ & $\begin{array}{c}\text { Mix C } \\
(\text { blank 2) }\end{array}$ & $\begin{array}{l}\text { Mix D } \\
(\text { medium) }\end{array}$ \\
\hline 1 м K-phosphate buffer pH 7.4 & 20 & 20 & 20 & 20 \\
\hline Distilled water & 143 & 155 & 155 & 160 \\
\hline $\begin{array}{l}\text { NADPH regenerating system solution } \\
\text { NADP }^{+}(26 \mathrm{~mm}) \\
\text { Glucose } 6 \text {-phosphate }(66 \mathrm{~mm}) \\
\text { Magnesium chloride }(66 \mathrm{~mm}) \\
\text { Glucose 6-phosphate dehydrogenase }(40 \text { units } / \mathrm{ml})\end{array}$ & 12 & 0 & 0 & 0 \\
\hline $\begin{array}{l}\text { Liver microsomes }(20 \mathrm{mg} / \mathrm{ml}) \\
\text { Incubation procedures }\end{array}$ & 5 & 5 & 5 & 0 \\
\hline Heat inactivation $\left(5 \min 80^{\circ} \mathrm{C}\right)$ & No & No & Yes & No \\
\hline$+20 \mu 1$ Py-Im polyamide 1035 or 1666 in distilled water & Yes & Yes & Yes & Yes \\
\hline Incubation $\left(37^{\circ} \mathrm{C}\right)$ & Yes & Yes & Yes & Yes \\
\hline
\end{tabular}

NADPH: reduced nicotinamide adenine dinucleotide phosphate. K: potassium.

Drug Administration Guidance for Industry (2001). ${ }^{6}$ Unchanged Py-Im polyamides 1035 and 1666 in rat urine and bile were determined with acceptable precision (coefficient of variance $(\mathrm{CV})<15 \%)$ and accuracy (relative error $(\mathrm{RE})< \pm 15 \%$ ). LLOQs for unchanged Py-Im polyamides 1035 and 1666 were experimentally defined as the lowest concentration of the calibration curve that could be measured with acceptable precision $(\mathrm{CV}<20 \%)$ and accuracy $(\mathrm{RE}< \pm 20 \%$ ). The LLOQs (with $\mathrm{CV}$ and $\mathrm{RE} \%$ ) for unchanged Py-Im polyamide 1035 were $1 \mu \mathrm{g} / \mathrm{ml}$ (with 1.19 and $13.36 \%$ ) for urine and $0.5 \mu \mathrm{g} / \mathrm{ml}$ (with 0.38 and $-0.15 \%$ ) for bile. The LLOQs (with CV and RE\%) for unchanged Py-Im polyamide 1666 were $1 \mu \mathrm{g} / \mathrm{ml}$ (with 1.93 and $7.27 \%$ ) for urine and also $1 \mu \mathrm{g} / \mathrm{ml}$ (with 1.94 and 1.97\%) for bile.

Urine and Bile Sampling Male Wistar rats (280 $400 \mathrm{~g})$ were obtained from Sankyo Labo Service Corporation (Tokyo, Japan). A cannula was inserted into the right jugular vein of the rats under anesthesia with pentobarbital sodium (Kyoritsu Seiyaku Corporation, Tokyo, Japan). In the urinary excretion study, each Py-Im polyamide dissolved in distilled water was administered intravenously to the jugular vein of the rats at a dose of $2.0 \mathrm{mg} / \mathrm{kg}$. Urine samples were collected after $1,3,5,8,12,24,36$, and $48 \mathrm{~h}$ using metabolic cages after dosing. In the biliary excretion study, a cannula was inserted into the bile duct of the rats under anesthesia with pentobarbital sodium. Each Py-Im polyamide was administered intravenously in the same manner as in the urinary excretion study. Biliary samples were collected $0.5,1,2.5,3,5,8,12$, 18 , and $24 \mathrm{~h}$ after dosing. The animal study was approved by the Animal Ethics Committee of the College of Pharmacy, Nihon University.

Metabolism by Liver Microsomes The possible metabolism of Py-Im polyamides 1035 and 1666 was investigated by determining the decrease in the concentration of the parent compound upon incubation with rat and human microsomes as described by Parnham et al. ${ }^{7)}$ Incubations with PyIm polyamides 1035 or $1666(1,10 \mu \mathrm{M})$ were performed at $37^{\circ} \mathrm{C}$ at a final volume of $200 \mu 1$ under four conditions (as shown in Table 1). All the incubations were performed in triplicate for 0,20 , and $60 \mathrm{~min}$, and the reactions were terminated by adding $400 \mu \mathrm{l}$ of ice-cold acetonitrile.

As a positive control for enzymatic activity in the micro- somes, midazolam hydroxylase (CYP3A) activity was determined within the same series of incubations. The incubations were performed in triplicate for 0,4 , and $8 \mathrm{~min}$, and the reactions were terminated by adding $200 \mu \mathrm{l}$ of ice-cold acetonitrile. Midazolam, 1'-hydroxymidazolam, and 4-hydroxymidazolam concentrations were determined using the modified HPLC procedure described by Boukhabza et al. ${ }^{8}$

Pharmacokinetic Modeling To describe the plasma concentration-time profile of Py-Im polyamide 1035 after intravenous administration, four pharmacokinetic models (i.e., one- and two-compartment models with the linear output compartment interpreted as the urine compartment and the linear and non-linear output compartments interpreted as the bile compartment) using the plasma concentration-time profile and cumulative urinary and biliary excretion data of PyIm polyamide 1035 were tested. A scheme of a two-compartment model with the linear output compartment interpreted as the urine compartment and the non-linear output compartment interpreted as the bile compartment is shown in Fig. 1A. ${ }^{3,9)}$ The equations used to describe the disposition of PyIm polyamide 1035 after intravenous administration are

$$
\begin{aligned}
\frac{d X_{\mathrm{C}}}{d t} & =C L_{\mathrm{D}} \cdot \frac{X_{\mathrm{P}}}{V_{\mathrm{P}}}-\left(C L_{\mathrm{D}}+C L_{\mathrm{R}}+\frac{V_{\mathrm{MAX}}}{K_{\mathrm{m}}+X_{\mathrm{C}} / V_{\mathrm{C}}}+C L_{\mathrm{ext}}\right) \cdot \frac{X_{\mathrm{C}}}{V_{\mathrm{C}}} \\
\frac{d X_{\mathrm{P}}}{d t} & =C L_{\mathrm{D}} \cdot \frac{X_{\mathrm{C}}}{V_{\mathrm{C}}}-C L_{\mathrm{D}} \cdot \frac{X_{\mathrm{P}}}{V_{\mathrm{P}}} \\
\frac{d X_{\mathrm{U}}}{d t} & =C L_{\mathrm{R}} \cdot \frac{X_{\mathrm{C}}}{V_{\mathrm{C}}} \\
\frac{d X_{\mathrm{B}}}{d t} & =\frac{V_{\mathrm{MAX}}}{K_{\mathrm{m}}+X_{\mathrm{C}} / V_{\mathrm{C}}} \cdot \frac{X_{\mathrm{C}}}{V_{\mathrm{C}}}
\end{aligned}
$$

where $X$ and $V$ are the amount and volume of distribution in the corresponding compartments designated by the subscripts $\mathrm{C}, \mathrm{P}, \mathrm{U}$, and $\mathrm{B}$ representing central, peripheral, urine, and bile compartments, $C L_{\mathrm{D}}$ is distribution clearance, $C L_{\mathrm{R}}$ is renal clearance, $C L_{\text {ext }}$ is clearance except renal and biliary clearance, $V_{\mathrm{MAX}}$ is maximum velocity for excretion into bile, and $K_{\mathrm{m}}$ is Michaelis constant for excretion into bile. The plasma concentration of Py-Im polyamide 1035 is defined as 


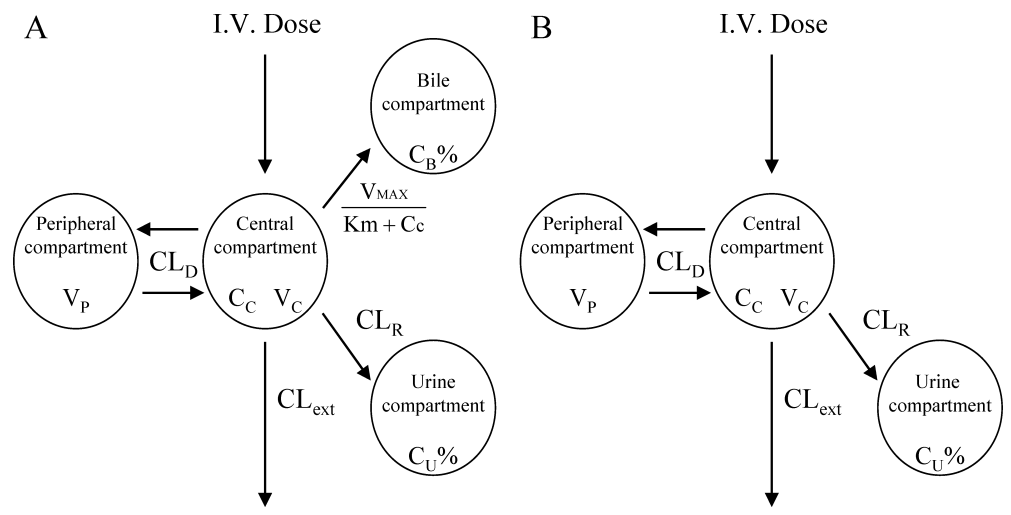

Fig. 1. Scheme of the Pharmacokinetic Model Describing the Disposition and Elimination of Py-Im Polyamides 1035 (A) and 1666 (B)

$C_{\mathrm{C}}$ is the plasma concentration of each Py-Im polyamide in the central compartment, and $V_{\mathrm{C}}$ and $V_{\mathrm{p}}$ are the volumes of distribution in the central and peripheral compartments, respectively. $C L_{\mathrm{D}}$ is distribution clearance, $C L_{\mathrm{R}}$ is renal clearance, $C L_{\text {ext }}$ is clearance except renal and biliary clearance, $V_{\mathrm{MAX}}$ is maximum velocity for excretion into bile, $K_{\mathrm{m}}$ is Michaelis constant. $C_{\mathrm{U}} \%$ and $C_{\mathrm{B}} \%$ represent the cumulative urinary and biliary excretions (percentage of administered dose), respectively.

$$
C_{\mathrm{C}}=X_{\mathrm{C}} / V_{\mathrm{C}}
$$

where $C_{\mathrm{C}}$ represents the plasma concentration of Py-Im polyamide 1035 . The cumulative urinary and biliary excretions of Py-Im polyamide 1035 are defined as

$$
\begin{aligned}
& C_{\mathrm{U}} \%=\frac{X_{\mathrm{U}}}{\text { Dose }} \cdot 100 \\
& C_{\mathrm{B}} \%=\frac{X_{\mathrm{B}}}{\text { Dose }} \cdot 100
\end{aligned}
$$

where $C_{\mathrm{U}} \%$ and $C_{\mathrm{B}} \%$ represent the cumulative urinary and biliary excretions (percentage of administered dose), respectively. To describe the plasma concentration-time profile of Py-Im polyamide 1666, two pharmacokinetic models (i.e., one- and two-compartment models with the linear output compartment interpreted as the urine compartment) using the plasma concentration-time profile and cumulative urinary excretions of Py-Im polyamide 1666 were tested. A scheme of the two-compartment model with the linear output compartment interpreted as the urine compartment is shown in Fig. 1B. The following equations describe the disposition of Py-Im polyamide 1666 after intravenous administration.

$$
\begin{aligned}
& \frac{d X_{\mathrm{C}}}{d t}=C L_{\mathrm{D}} \cdot \frac{X_{\mathrm{P}}}{V_{\mathrm{P}}}-\left(C L_{\mathrm{D}}+C L_{\mathrm{R}}+C L_{\text {ext }}\right) \cdot \frac{X_{\mathrm{C}}}{V_{\mathrm{C}}} \\
& \frac{d X_{\mathrm{p}}}{d t}=C L_{\mathrm{D}} \cdot \frac{X_{\mathrm{C}}}{V_{\mathrm{C}}}-C L_{\mathrm{D}} \cdot \frac{X_{\mathrm{P}}}{V_{\mathrm{P}}} \\
& \frac{d X_{\mathrm{U}}}{d t}=C L_{\mathrm{R}} \cdot \frac{X_{\mathrm{C}}}{V_{\mathrm{C}}} \\
& C_{\mathrm{C}}=X_{\mathrm{C}} / V_{\mathrm{C}} \\
& C_{\mathrm{U}} \%=\frac{X_{\mathrm{U}}}{\text { Dose }} \cdot 100
\end{aligned}
$$

The residual error models of the plasma concentration of Py-Im polyamide 1035 or 1666 and cumulative urinary and biliary excretions used were

$$
Y_{\text {obs, }}=Y_{\text {pred }, \mathrm{p}} \cdot\left(1+\varepsilon_{1}\right)
$$

$$
\begin{aligned}
& Y_{\text {obs, } \mathrm{u}}=Y_{\text {pred, } \mathrm{u}}+\varepsilon_{2} \\
& Y_{\text {obs, } \mathrm{b}}=Y_{\text {pred, } \mathrm{b}}+\varepsilon_{3}
\end{aligned}
$$

where $Y_{\mathrm{obs}, \mathrm{p}}, Y_{\mathrm{obs}, \mathrm{u}}$, and $Y_{\mathrm{obs}, \mathrm{b}}$ are the measured plasma concentration of Py-Im polyamide 1035 or 1666 and the measured cumulative urinary and biliary excretions (percentage of

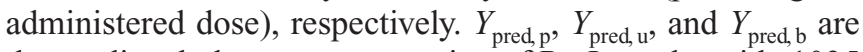
the predicted plasma concentration of Py-Im polyamide 1035 or 1666 and the predicted cumulative urinary and biliary excretions (percentage of administered dose) in accordance with the model, respectively. $\varepsilon_{1}, \varepsilon_{2}$, and $\varepsilon_{3}$ are the normally distributed error terms with means of zero and variances of $\sigma_{1}^{2}, \sigma_{2}^{2}$, and $\sigma_{3}^{2}$, respectively. The residual error model of the plasma concentration was assumed to be the proportional error model because the plasma concentration was measured by HPLC. The model of the cumulative urinary and biliary excretions was assumed to be the additive error model because the percentage of the administered dose was calculated from the urine and bile concentrations, the urine and bile volumes, and the administered dose.

The parameters were estimated by the naïve pooled data method in which all the data from all the rats are considered as arising from one unique rat. ${ }^{10)}$ Initial parameter estimates were obtained by referring to the values of moment analysis and their cumulative urinary and biliary excretion ratios. All the data sets were estimated and simulated using the computer software package NONMEM ver. 6 (ICON development solutions, MD, U.S.A. $)^{9,11)}$ in conjunction with Intel Visual Fortran Compiler ver. 10 and Wings for NONMEM. ${ }^{12}$ ) To obtain 2.5 th, 50th, and 97.5 th percentiles of the model estimations, 10000 simulations were performed using the estimated model parameters, variability in the estimated parameters, and residual variability of the data. The plasma concentration-time profiles of Py-Im polyamides 1035 and 1666 were obtained from the study of Fukasawa et al. ${ }^{4)}$ The excluded plasma concentrations below LLOQ have been shown to adversely affect the bias and precision of parameter estimates. ${ }^{1,2)}$ The detected concentration below LLOQ of HPLC method was used in the PK modeling. The choice of model was based on model fitting criteria such as visual inspection of the fitted curves, objective function value of NONMEM (OFV), and CV\% of the parameter estimates. ${ }^{11,13)}$ 


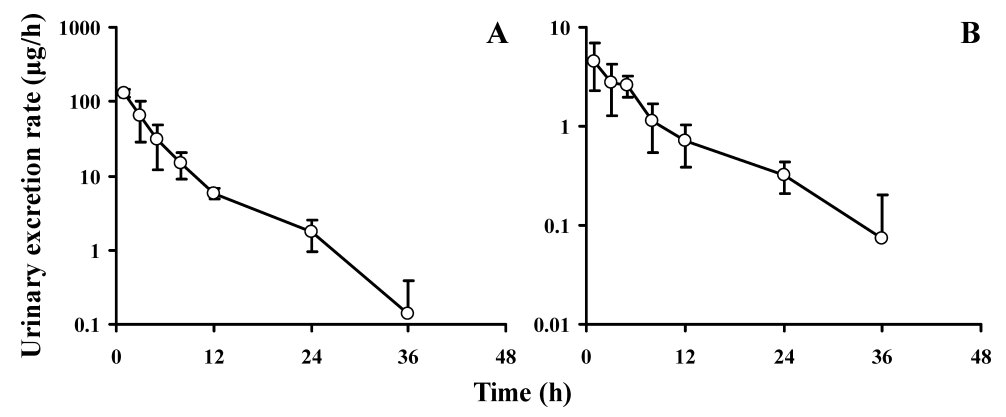

Fig. 2. Urinary Excretion Rate versus Time Profile of Py-Im Polyamides 1035 (A) and 1666 (B) in Rats

Each Py-Im polyamide dissolved in distilled water was administered intravenously to the jugular vein of the rats at a dose of $2.0 \mathrm{mg} / \mathrm{kg}$. Urine samples were collected after 1,3 , $5,8,12,24,36$, and $48 \mathrm{~h}$ using metabolic cages after dosing. Each data point represents the mean \pm S.D. of three rats.

\section{RESULTS}

Urinary and Biliary Excretions The urinary and biliary excretion rate-time profiles are shown in Figs. 2 and 3, respectively. The urinary excretion rates of Py-Im polyamides 1035 and 1666 showed linear elimination. The biliary excretion rate of Py-Im polyamide 1035 showed saturation at the early period. The biliary excretion of Py-Im polyamide 1666 was not detected. The cumulative urinary excretions of PyIm polyamides 1035 and 1666 at $48 \mathrm{~h}$ were $72.4 \pm 11.6$ and $4.8 \pm 0.5 \%$ (mean \pm S.D., $n=3$ ) of the administered dose, respectively. The cumulative biliary excretion of Py-Im polyamide 1035 at $24 \mathrm{~h}$ was $4.3 \pm 0.4 \%(n=4)$ of the administered dose. No peaks of metabolites were detected for all the samples.

Metabolism by Liver Microsomes The metabolism of Py-Im polyamides 1035 and $1666(1,10 \mu \mathrm{M})$ was investigated using rat and human liver microsomes. The peaks of their metabolites and the decreases in the Py-Im polyamide concentrations with incubation time were not detected under the experimental conditions. The average activities of midazolam 1'-hydroxylation for rat and human microsomes were 0.25 and $0.21 \mathrm{nmol} / \mathrm{min} / \mathrm{mg}$ protein, respectively. The average activities of midazolam 4-hydroxylation for rat and human microsomes were 5.46 and $0.33 \mathrm{nmol} / \mathrm{min} / \mathrm{mg}$ protein, respectively.

Pharmacokinetic Modeling The results obtained using the different models tested in this study are shown in Table 2. The plasma concentration and cumulative urinary and biliary excretion-time profiles after intravenous administration of Py-Im polyamide 1035 were described using a two-compartment model with the linear output compartment interpreted as the urine compartment and the non-linear output compartment interpreted as the bile compartment, as shown in Fig. 4. The plasma concentration and cumulative urinary excretiontime profiles after intravenous administration of Py-Im polyamide 1666 were described using a two-compartment model with the linear output compartment interpreted as the urine compartment, as shown in Fig. 5. Good agreement was demonstrated between the observed and predicted values of Py-Im polyamides 1035 and 1666 . In this figure, the 50th percentiles and 2.5th and 97.5th percentiles of the modelbased prediction for plasma concentrations and cumulative urinary and biliary excretions are presented together with the observed value. This figure provides evidence that the developed model is appropriate for describing the plasma concen-

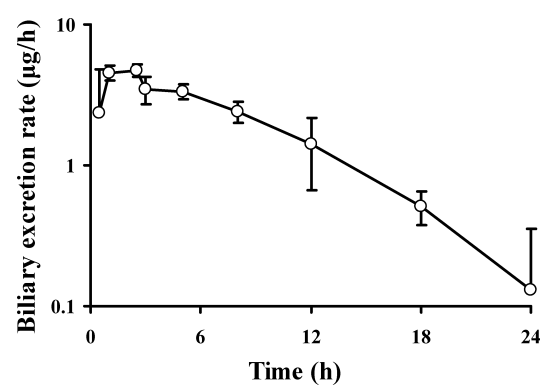

Fig. 3. Biliary Excretion Rate versus Time Profile of Py-Im Polyamide 1035 in Rats

Py-Im polyamide 1035 dissolved in distilled water was administered intravenously to the jugular vein of the rats at a dose of $2.0 \mathrm{mg} / \mathrm{kg}$. Biliary samples were collected $0.5,1$, $2.5,3,5,8,12,18$, and $24 \mathrm{~h}$ after dosing. Each data point represents the mean \pm S.D. of four rats.

Table 2. Summary of Model Building

\begin{tabular}{|c|c|c|c|}
\hline \multicolumn{3}{|c|}{ PK model } & \multirow{2}{*}{ OFV } \\
\hline Plasma & Urine & Bile & \\
\hline \multicolumn{4}{|l|}{ Py-Im polyamide 1035} \\
\hline One-compartment & Linear output & Linear output & 166.087 \\
\hline One-compartment & Linear output & Non-linear output & 86.351 \\
\hline Two-compartment & Linear output & Linear output & 110.391 \\
\hline Two-compartment & Linear output & Non-linear output & 44.009 \\
\hline \multicolumn{4}{|l|}{ Py-Im polyamide 1666} \\
\hline One-compartment & Linear output & - & -16.021 \\
\hline Two-compartment & Linear output & - & -32.494 \\
\hline
\end{tabular}

tration and cumulative urinary and biliary excretion-time profiles after intravenous administration of Py-Im polyamides 1035 and 1666. The PK parameter estimates are summarized in Table 3.

\section{DISCUSSION}

In this study, a two-compartment model with linear and non-linear output compartments was developed using plasma concentration and cumulative urinary and biliary excretion data. The PK model describing first-order excretion into urine or a saturation of renal tubular secretion has already been reported. ${ }^{14)}$ There is no reported PK model describing the plasma concentration-time profiles using simultaneous first-order excretion into urine and a saturation of secretion into bile. This proposed PK model can be used to more accurately predict the plasma concentration-time profiles under 


\section{LLOQ.}

Py-Im polyamides are synthesized by combining $\mathrm{N}$ methylpyrrole and $N$-methylimidazole amino acids. ${ }^{15-17)}$ The various combinations have enabled the recognition and binding of Py-Im polyamides to specific nucleotide sequences of double-helical DNA with high affinity. ${ }^{17-20)}$ In this study, two types of Py-Im polyamide, 1035 and 1666, with molecular weights of 1035.12 and 1665.78 , respectively, were used. Py-Im polyamide 1666 with a hairpin structure of $\gamma$-butyric

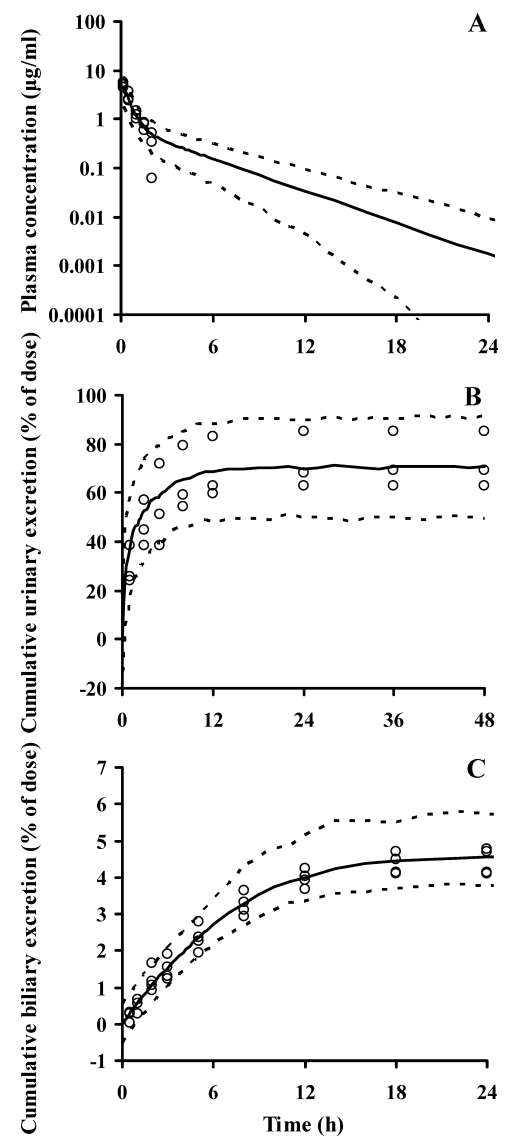

Fig. 4. Plasma Concentration-Time Profile (A), Cumulative Urinary Excretion (B), and Cumulative Biliary Excretion (C) of Py-Im Polyamide 1035 after Intravenous Administration of $2.0 \mathrm{mg} / \mathrm{kg}$ to Rats

Each data point represents observed data from three (for plasma and urine) and four rats (for bile). The solid line indicates 50th percentiles, and the broken lines represent 2.5 th and 97.5 th percentiles from model estimations of 10000 simulations. acid is designed to bind to the activator protein-1 (AP-1) binding site of the rat transforming growth factor- $\beta 1$ (TGF$\beta 1$ ) promoter, whereas Py-Im polyamide 1035 also with a hairpin structure is designed for comparing with other types of Py-Im polyamide with hairpin structure and higher molecular weight. Py-Im polyamide 1666 may become a novel gene-silencing agent for the TGF- $\beta 1$-associated diseases including progressive renal diseases because it was found to reduce the amount of urinary protein and albumin in Dahl-S rats after intravenous injection of $1 \mathrm{mg}$ Py-Im polyamide 1666 every 2 days for 2 weeks. ${ }^{21)}$ However, the elimination pathway of unchanged Py-Im polyamides 1035 and 1666 in rats after intravenous administration and their metabolism in vivo and in vitro have not been elucidated.

Drugs are usually eliminated from the body into urine and/or bile. ${ }^{22,23)}$ Therefore, Py-Im polyamides 1035 and 1666 can be characterized by determining their urinary and biliary excretions. The urinary excretions of Py-Im polyamides 1035 and 1666, which were determined by HPLC, were high (72.4\%) and low (4.8\%), respectively, at $48 \mathrm{~h}$. The biliary excretion of Py-Im polyamide 1666 was not detected but that of Py-Im polyamide 1035 was $4.3 \%$ at $24 \mathrm{~h}$. These observations indicated that unchanged Py-Im polyamides 1035 and 1666 have been slowly eliminated from the body. Particularly, it is considered that most of the Py-Im polyamide 1666 remains in the body and that it is distributed in deep compartments (i.e., in organs such as liver, kidney, lung, and brain) or in multiple compartments.

The oxidation of Py-Im polyamides 1035 and 1666 mediated by $\mathrm{P} 450$ was not detected using rat and human microsomes. Moreover, Py-Im polyamides 1035 and 1666 were also not detected in the rat urine and bile. The metabolism of

Table 3. Pharmacokinetic Parameter Estimates

\begin{tabular}{lccccc}
\hline \hline & \multicolumn{2}{c}{ Py-Im polyamide 1035 } & & \multicolumn{2}{c}{ Py-Im polyamide 1666 } \\
\cline { 2 - 3 } \cline { 5 - 6 } & Value & CV\% & & Value & CV\% \\
& & & & & \\
\cline { 5 - 6 }$V_{\mathrm{C}}(\mathrm{ml} / \mathrm{kg})$ & 310 & 11.9 & & 599 & 15.2 \\
$V_{\mathrm{P}}(\mathrm{ml} / \mathrm{kg})$ & 591 & 23.2 & & 1810 & 36.5 \\
$C L_{\mathrm{D}}(\mathrm{ml} / \mathrm{min} / \mathrm{kg})$ & 4.38 & 24.4 & & 12.7 & 25.4 \\
$C L_{\mathrm{R}}(\mathrm{ml} / \mathrm{min} / \mathrm{kg})$ & 4.32 & 10.2 & & 0.263 & 15.1 \\
$C L_{\text {ext }}(\mathrm{ml} / \mathrm{min} / \mathrm{kg})$ & 1.55 & 18.2 & & 5.15 & 14.4 \\
$V_{\mathrm{MAX}}(\mu \mathrm{gg} / \mathrm{min} / \mathrm{kg})$ & 0.205 & 12.0 & & - & - \\
$K_{\mathrm{m}}(\mu \mathrm{g} / \mathrm{ml})$ & 0.145 & 57.6 & & - & - \\
\hline
\end{tabular}

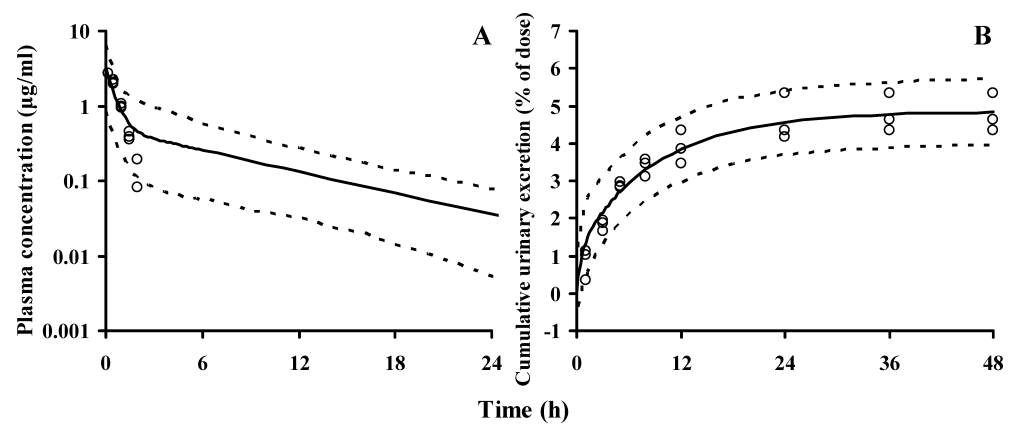

Fig. 5. Plasma Concentration-Time Profile (A) and Cumulative Urinary Excretion (B) of Py-Im Polyamide 1666 after Intravenous Administration of $2.0 \mathrm{mg} / \mathrm{kg}$ to Rats

Each data point represents observed data from three rats (for plasma and urine). The solid line indicates 50th percentiles, and the broken lines represent 2.5 th and 97.5 th percentiles from model estimations of 10000 simulations. 
Py-Im polyamides 1035 and 1666 mediated by uridine 5'diphosphate (UDP)-glucuronosyltransferase and sulfotransferase in rat microsomes and cytosol was also not detected (data not shown). These observations indicate that Py-Im polyamide 1035 is not metabolized but most of it is excreted unchanged into the urine and bile, whereas Py-Im polyamide 1666 also is not metabolized but most of it may be distributed extensively to organs and tissues. Further studies are required to clarify its metabolism by other enzymes or its tissue distribution. To the best of our knowledge, there is no information on the elimination and metabolism of unchanged Py-Im polyamides in rat.

The differences in the molecular weights of compounds affect their eliminations. ${ }^{24)}$ The molecular weight thresholds for the excretion of organic cations into rat bile were found to be in the range of $200 \pm 50$ for monovalent organic cations and $500-600$ for bivalent organic cations. ${ }^{25,26)}$ Py-Im polyamide of 1422.51 molecular weight was excreted at $2 \%$ into rat urine $24 \mathrm{~h}$ after administration and not detected in rat bile (data not shown). These findings suggested the possibility that Py-Im polyamides with high molecular weights tend to be poorly excreted in both rat urine and bile, whereas those with molecular weights less than that of Py-Im polyamide 1035 can be readily eliminated. As described above, the differences in the elimination pathway between Py-Im polyamides 1035 and 1666 may be attributed to the differences in their molecular weights.

When Py-Im polyamides 1035 and 1666 were administered orally to rats, the absorption from the intestine was not detected by HPLC method. The rat plasma concentrations of Py-Im polyamides 1035 and 1666 after intravenous administration at $2.0 \mathrm{mg} / \mathrm{kg}$ were less than approximately $1 \mu \mathrm{g} / \mathrm{ml}$ after $2 \mathrm{~h}^{4)}$ The validated HPLC cannot be used to determine less than $1 \mu \mathrm{g} / \mathrm{ml}^{4}{ }^{4}$ Therefore, we developed a model to simulate the plasma concentration-time profiles under LLOQ of HPLC using the excretion data.

The main objective is to develop a PK model that can describe the PK profiles of Py-Im polyamides 1035 and 1666 including excretion data such as the urinary and biliary excretions of unchanged drug and metabolites. Py-Im polyamides 1035 and 1666 were not metabolized, and unchanged Py-Im polyamide 1666 was not detected in the bile. Moreover, Py-Im polyamides 1035 and 1666 were not absorbed via the intestine. Therefore, we assumed that the metabolism and intestinal circulation of these compounds are negligible, and thus we used the plasma concentration and urinary and biliary excretion data for the modeling of unchanged Py-Im polyamide 1035, and for unchanged Py-Im polyamide 1666 , plasma concentration and urinary excretion data were used.

In the modeling for Py-Im polyamide 1035, a one-compartment model using only plasma data was used to compare with the final PK model that includes rat plasma, urine, and bile data. The plasma Py-Im polyamide 1035 concentration predicted using the one-compartment model was almost reduced to zero $8 \mathrm{~h}$ after administration (data not shown). The result obtained using the model was different from the observed urine and bile data because Py-Im polyamide 1035 was excreted into urine and bile until at least 36 and $18 \mathrm{~h}$, respectively, after administration. Thus, the one-compartment model could not adequately describe a PK profile; therefore, a PK model was developed using the plasma concentration and urinary and biliary excretion data. The parameters were estimated by the naïve pooled data method because the plasma, urine, and bile data were obtained from different rats. The PK model was tested using a two-compartment model with two linear output compartments for urine and bile. The described cumulative urinary and biliary excretion data were different from the observed urinary and biliary excretion data. From the biliary excretion rate-time profile, a constant elimination at an early period was observed (Fig. 3). The biliary excretion is considered to be saturated. Therefore, the biliary output compartment model was changed from linear excretion to saturated non-linear excretion of bile. Finally, the changed PK model could adequately describe all the data, and the plasma concentration-time profile under LLOQ could also be described better using both linear and non-linear compartments than using plasma data only. The plasma concentration-time profile of Py-Im polyamide 1666 was adequately described using the two-compartment model built with the linear output compartment interpreted as the urine compartment. Moreover, the PK model built using saturated biliary excretion data can be used to simultaneously assess the effects of saturations and competitive inhibition of transporters because biliary excretion is associated with various transporters. If the compounds could be metabolized, the plasma concentration-time profile below LLOQ could be described using the plasma and excretion data of the metabolite. The PK parameter estimation using the plasma concentration data except those below LLOQ together with the urine concentration data could be more accurate than using only the plasma concentration data except those below LLOQ in a simulation study. ${ }^{3)}$ Therefore, this approach can be applied to not only Py-Im polyamides 1035 and 1666 used in this study but also to various compounds.

In conclusion, the PK model describing plasma Py-Im polyamide concentrations was developed using urinary and biliary excretion data. This is the first report showing that, in rats, the PK model can be used to describe the plasma concentration-time profiles using the linear output compartment interpreted as the urine compartment and the non-linear output compartment interpreted as the bile compartment. Moreover, the possibility that the undetectable plasma concentration-time profile can be described using urinary and biliary excretion data is considered. If the metabolism and intestinal circulation of drugs are negligible, this PK model can provide a more accurate prediction of the plasma concentration profiles under LLOQ.

Acknowledgments We thank Bun Igarashi, Takafumi Nagashima, and Yoshimi Takasaki for their help. This work was partially supported by a grant from "Academic Frontier" Project for Private Universities from the Ministry of Education, Culture, Sports, Science and Technology of Japan (2006-2010).

\section{REFERENCES}

1) Duval V., Karlsson M. O., Pharm. Res., 19, 1835-1840 (2002).

2) Beal S. L., J. Pharmacokinet. Pharmacodyn., 28, 481-504 (2001).

3) Yoshihara K., 〈http://pagja.org/23rd_PAGJA_2007/23rdPPK/P12_ Yoshihara.pdf $\rangle$, The 23rd population approach group in Japan, p. 12, July (2007). 
4) Fukasawa A., Nagashima T., Aoyama T., Fukuda N., Matsuda H., Ueno T., Sugiyama H., Nagase H., Matsumoto Y., J. Chromatogr. B, Anal. Technol. Biomed. Life Sci., 859, 272-275 (2007).

5) Wieser M. E., Pure Appl. Chem., 78, 2051-2066 (2006).

6) U.S. Department of Health and Human Services, Food and Drug Administration, Center for Drug Evaluation and Research (CDER), Center for Veterinary Medicine (CVM), 〈http://www.fda.gov/cder/ Guidance/4252fnl.pdf), May 2001.

7) Parnham M. J., Bogaards J. J., Schrander F., Schut M. W., Oreskovic K., Mildner B., Biopharm. Drug Dispos., 26, 27-33 (2005).

8) Boukhabza A., Lugnier A. A., Kintz P., Mangin P., J. Anal. Toxicol., 15, 319-322 (1991).

9) Beal S. L., Sheiner L. B., “NONMEM Users' Guide,” NONMEM Project Group, University of California, San Francisco, 1988.

10) Ette E. I., Williams P. J., Ann. Pharmacother, 38, 1907-1915 (2004).

11) Matsumoto Y., Fujita T., Ishida Y., Shimizu M., Kakuo H., Yamashita K., Majima M., Kumagai Y., Biol. Pharm. Bull., 28, 1455-1461 (2005).

12) Holford N., 〈http://wfn.sourceforge.net/>, Wings for NONMEM, 12 December 2008.

13) Hazra A., Pyszczynski N., DuBois D. C., Almon R. R., Jusko W. J., Biopharm. Drug Dispos., 28, 263-273 (2007).
14) Thompson G. A., Toothaker R. D., Pharm. Res., 21, 781 -784 (2004).

15) White S., Baird E. E., Dervan P. B., Chem. Biol., 4, 569-578 (1997).

16) Trauger J. W., Baird E. E., Dervan P. B., Nature (London), 382, 559561 (1996).

17) Dervan P. B., Bioorg. Med. Chem., 9, 2215-2235 (2001).

18) Murty M. S., Sugiyama H., Biol. Pharm. Bull., 27, $468-474$ (2004).

19) Swalley S. E., Baird E. E., Dervan P. B., Chemistry, 3, 1600-1607 (1997).

20) Turner J. M., Swalley S. E., Baird E. E., Dervan P. B., J. Am. Chem. Soc., 120, 6219-6226 (1998).

21) Matsuda H., Fukuda N., Ueno T., Tahira Y., Ayame H., Zhang W., Bando T., Sugiyama H., Saito S., Matsumoto K., Mugishima H., Serie K., J. Am. Soc. Nephrol., 17, 422-432 (2006).

22) Ullrich K. J., J. Membr. Biol., 158, 95-107 (1997).

23) van Montfoort J. E., Hagenbuch B., Groothuis G. M., Koepsell H., Meier P. J., Meijer D. K., Curr. Drug Metab., 4, 185-211 (2003).

24) Hirom P. C., Millburn P., Smith R. L., Xenobiotica, 6, 55-64 (1976).

25) Hughes R. D., Millburn P., Williams R. T., Biochem. J., 136, 967-978 (1973).

26) Hughes R. D., Millburn P., Williams R. T., Biochem. J., 136, 979-984 (1973). 